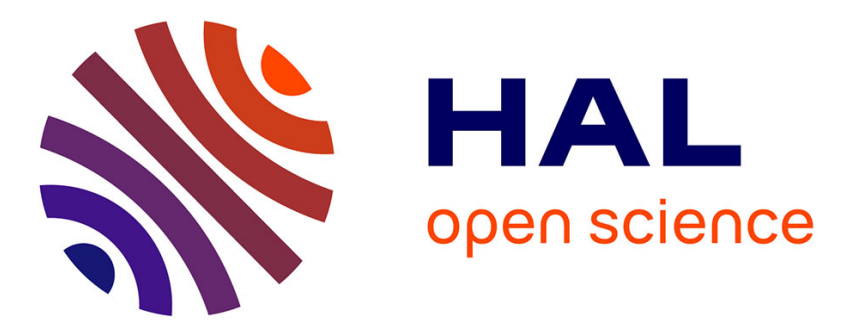

\title{
Experimental Measurements of Electron-Bunch Trains in a Laser-Plasma Accelerator
}

\author{
O Lundh, C Rechatin, J Lim, Victor Malka, Jérôme Faure
}

\section{To cite this version:}

O Lundh, C Rechatin, J Lim, Victor Malka, Jérôme Faure. Experimental Measurements of ElectronBunch Trains in a Laser-Plasma Accelerator. Physical Review Letters, 2013, 110, pp.065005. 10.1103/PhysRevLett.110.065005 . hal-01159044

\section{HAL Id: hal-01159044 \\ https://hal-ensta-paris.archives-ouvertes.fr/hal-01159044}

Submitted on 2 Jun 2015

HAL is a multi-disciplinary open access archive for the deposit and dissemination of scientific research documents, whether they are published or not. The documents may come from teaching and research institutions in France or abroad, or from public or private research centers.
L'archive ouverte pluridisciplinaire HAL, est destinée au dépôt et à la diffusion de documents scientifiques de niveau recherche, publiés ou non, émanant des établissements d'enseignement et de recherche français ou étrangers, des laboratoires publics ou privés. 


\title{
Experimental measurements of electron bunch trains in a laser-plasma accelerator
}

\author{
O. Lundh, ${ }^{1,2, *}$ C. Rechatin, ${ }^{2}$ J. Lim,${ }^{2}$ V. Malka, ${ }^{2}$ and J. Faure ${ }^{2, \dagger}$ \\ ${ }^{1}$ Department of Physics, Lund University, P.O. Box 118, S-22100 Lund, Sweden \\ ${ }^{2}$ Laboratoire d'Optique Appliquée, ENSTA, École Polytechnique, CNRS, 91761 Palaiseau, France
}

\begin{abstract}
Spectral measurements of visible coherent transition radiation produced by a laser-plasma accelerated electron beam are reported. The significant periodic modulations that are observed in the spectrum result from interference of transition radiation produced by multiple bunches of electrons. A Fourier analysis of the spectral interference fringes reveals that electrons are injected and accelerated in multiple plasma wave periods, up to at least ten periods behind the laser pulse. The bunch separation scales with the plasma wavelength when the plasma density is changed over a wide range. An analysis of the spectral fringe visibility indicates that the first bunch contains most of the charge.
\end{abstract}

In a laser wakefield accelerator (LWFA) [1], an intense and ultrashort laser pulse drives a relativistic plasma wave, or wakefield, which can be used to accelerate electrons to high energies in a short distance. With accelerating gradients in excess of $100 \mathrm{GV} / \mathrm{m}$, electron bunches in the $100 \mathrm{MeV}-1 \mathrm{GeV}$ range are now produced in $\mathrm{mm}$ distances, with few percent energy spreads and charges of 10's of picocoulombs [2,3]. These electron bunches also have the characteristic feature of having femtosecond duration and kA peak current [4], which make them good candidates as a compact electron source for a Free Electron Laser. This has triggered a large number of experimental studies aiming at characterizing the temporal structure of these laser-plasma produced electron bunches. The technique of choice has been Coherent Transition Radiation (CTR) which can give insights on the temporal spread of the electron bunches [5]. The first studies have focused on measuring CTR in the THz region, showing that the bunches were sub-100 fs [6]. More recently, several experiments have shown that the electron bunches can be as short as a few femtoseconds in duration $[4,7]$ but these works focussed on the electron bunch contained in the first bucket of the plasma wave. Few studies have considered the fact that the electron beam can be composed of several beamlets although some publications suggest that it can occur in certain cases $[8,9]$. Here, we present a detailed experimental study showing that several short electron bunches can be injected and efficiently accelerated in multiple plasma wave periods, thus forming an electron bunch train.

We use spectral measurements of CTR to diagnose the temporal distribution of electrons. CTR is emitted when the electron bunch passes an interface between two media, e.g. a metallic foil and a vacuum. For a monoenergetic electron beam, the angular radiation field is a hollow cone with half opening angle $\theta=1 / \gamma$. The spectral radiation field at frequency $\omega$ and observation angle $\theta$, is given by $[10-12]$

$$
\frac{d^{2} W}{d \omega d \Omega}=\left[N+N^{2} F(\omega, \theta)\right] \frac{d^{2} w}{d \omega d \Omega},
$$

where $\frac{d^{2} w}{d \omega d \Omega}$ is the transition radiation spectrum emitted by a single electron, $\Omega$ is the solid angle and $N$ is the number of electrons in the bunch. The form factor $F(\omega, \theta)=\left|\int f(\vec{x}) \exp (-i \vec{k} \cdot \vec{x}) d^{3} \vec{x}\right|^{2}$ is the square amplitude of the Fourier transform of the normalized electron bunch distribution, $f(\vec{x})$, and $\vec{k}$ is the wavevector in the direction of observation. The radiation is said to be coherent if the second term in Eq. (1) dominates. This typically occurs only if the electron bunch length is comparable to or less than the observed radiation wavelength. In this case, electrons radiate in phase and the radiation is added coherently.

The experiment was performed using the $10 \mathrm{~Hz}$, multiterawatt, Ti:Sapphire laser system in "Salle Jaune" at Laboratoire d'Optique Appliquée. The system delivers $30 \mathrm{fs}$ laser pulses containing $1 \mathrm{~J}$ of energy at $820 \mathrm{~nm}$. The experimental set-ups for producing electron beams was described in details in previous publications [4] (see also suppl. info. [13]). In this work, we have used two different injection techniques for producing an electron beam: self-injection (SI) and colliding pulse injection (CPI) $[14,15]$. In the case of SI, the non-linear spatio-temporal compression of the laser pulse leads to increased peak intensity, a steepening of the plasma wave and eventually transverse wavebreaking [16]. This mechanism can lead to quasi-monoenergetic electron beams [17-19]. For our experimental parameters, the beams obtained from self-injection had a large charge $(>90 \mathrm{pC})$ and large energy spreads (see Table I, see also suppl. info. [13]). In the case of CPI, the plasma wave was not driven to the breaking point. Instead, electrons were locally injected following the collision between the main pump pulse and a relatively weak, counter-propagating injection laser pulse $[2,20]$. This resulted in more stable beams with a narrower energy distribution but with lower charge $(\simeq 30 \mathrm{pC})$.

For CTR measurement, a $100 \mu \mathrm{m} \mathrm{Al}$ foil was placed on the path of the electron beam $15 \mathrm{~mm}$ from the exit of the gas jet. Forward CTR was generated at the rear surface of the foil which also served to stop the pump laser beam and prevent parasitic radiation from reaching the detection system. Before every shot the foil was translated to provide unexposed material for the electron beam.

The forward CTR propagated in the direction of the 

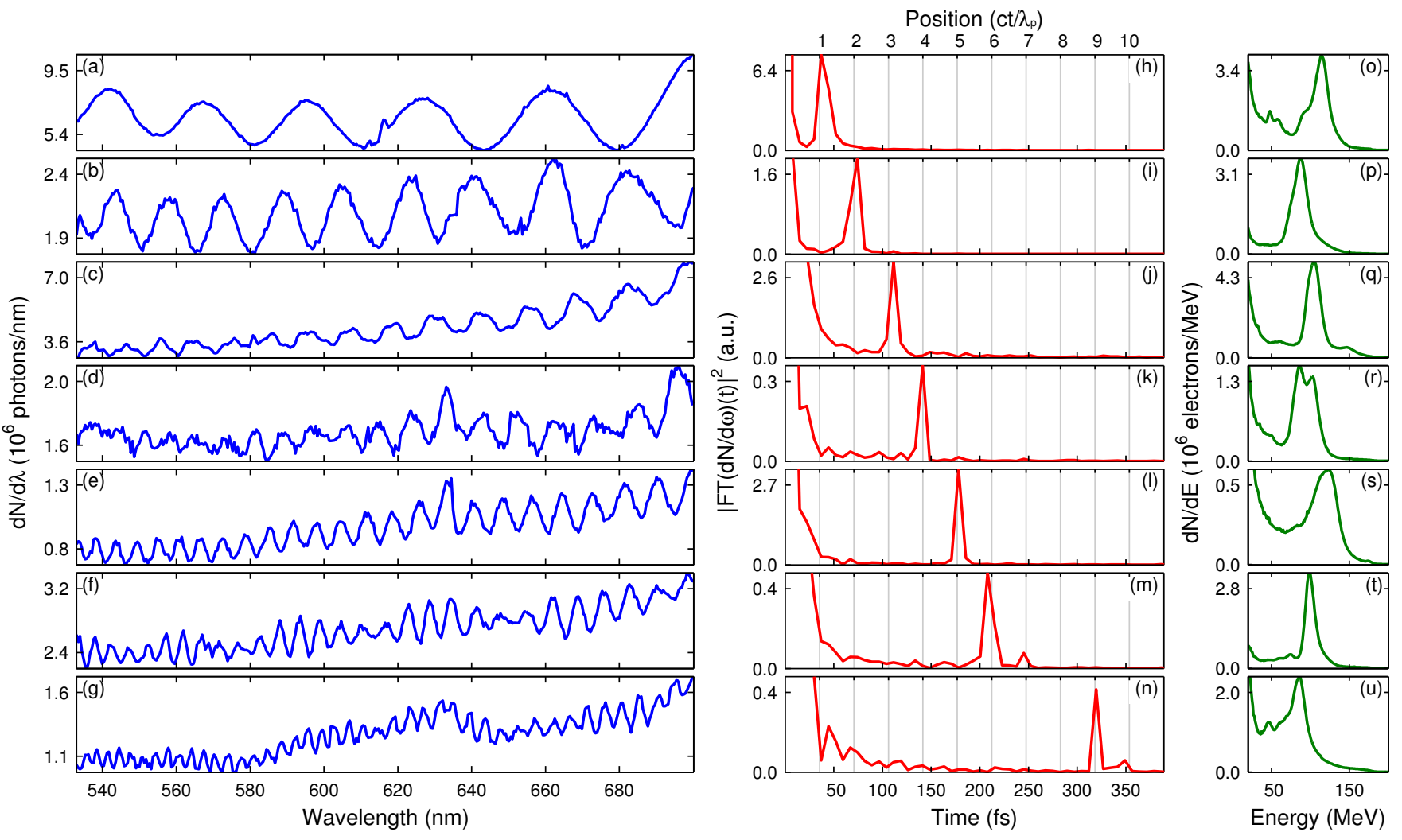

Figure 1. (Color online) Selected and sorted examples of sinusoidal CTR modulations, obtained using CPI for $n_{e}=10 \times 10^{18}$ $\mathrm{cm}^{-3}$ and $\lambda_{p}=10.6 \mu \mathrm{m}$. Three panels on each row represent one measurement. (a-g): CTR spectra. (h-n): Square amplitude of the Fourier transform of the CTR spectra. (o-u): Corresponding electron spectra.

electron beam and was collected and collimated outside the interaction chamber by a $1.5 \mathrm{~m}$-focal-length spherical silver mirror. The CTR spectrum was diagnosed using an imaging Czerny-Turner spectrometer, fitted with a 16bit CCD camera. The setup was calibrated to give the absolute number of photons per wavelength interval.

With this setup, electron- and optical spectra were measured simultaneously. Spectrally broadband radiation, originating from a well defined point source on the optical axis, was observed, but only in coincidence with the generation of an electron beam. The intensity fluctuated from shot-to-shot, but increased with electron beam charge. The optical transition radiation was found to be coherent because the intensity level was more than 3 orders of magnitude higher than the expected level of incoherent transition radiation (see suppl. info. [13]). Interestingly and unexpectedly, the measured CTR spectrum often showed significant spectral modulation fringes. The modulations were either purely sinusoidal, as shown in Fig. 1, or more complex as shown in Fig. 2. We have found that, in fact, these modulations are spectral interference fringes that contain very interesting temporal information about the electron beam.

Suppose that two electron bunches arrive at the radiator with some time difference $T$. Each bunch produces a continuous spectrum but, since the time of emission is slightly different, interference leads to a spectrum modulated by $g(\omega)=\cos (\omega T)$. The period of the modulation is directly related to the temporal bunch separation.

It is straightforward to calculate the separation between two bunches. For one oscillation, $\Delta \omega=\omega_{1}-\omega_{2}=$ $2 \pi / T$, which, if expressed in wavelengths, gives the bunch separation $L=c T \simeq \lambda_{o}^{2} / \Delta \lambda$. Applied to Fig. 1a, this simple estimate gives $L=0.600^{2} / 0.033=10.9 \mu \mathrm{m}$. This is remarkably close to the plasma wavelength at the density at which the measurements were made $\left(\lambda_{p}=10.6\right.$ $\mu \mathrm{m}$ at $\left.n_{e}=10 \times 10^{18} \mathrm{~cm}^{-3}\right)$. Similarly, Fig. 1e gives $L=54 \mu \mathrm{m}=5.1 \lambda_{p}$. These estimates, however simple, are strong indications that the spectral interference fringes originate from electrons which have been trapped and accelerated in multiple plasma wave periods.

Figure 1 shows selected and sorted examples of CTR spectra with sinusoidal modulations. The central panels in Fig. 1 show the square amplitude of the Fourier transform of the modulated CTR spectra. The Fourier transform here goes from the frequency domain to the time domain. For clarity, the temporal axis has also been multiplied by $c$ and divided by $\lambda_{p}$. Interestingly, the positions of the peaks in the Fourier transform coincide with multiples of the plasma wavelength, as marked by the vertical 

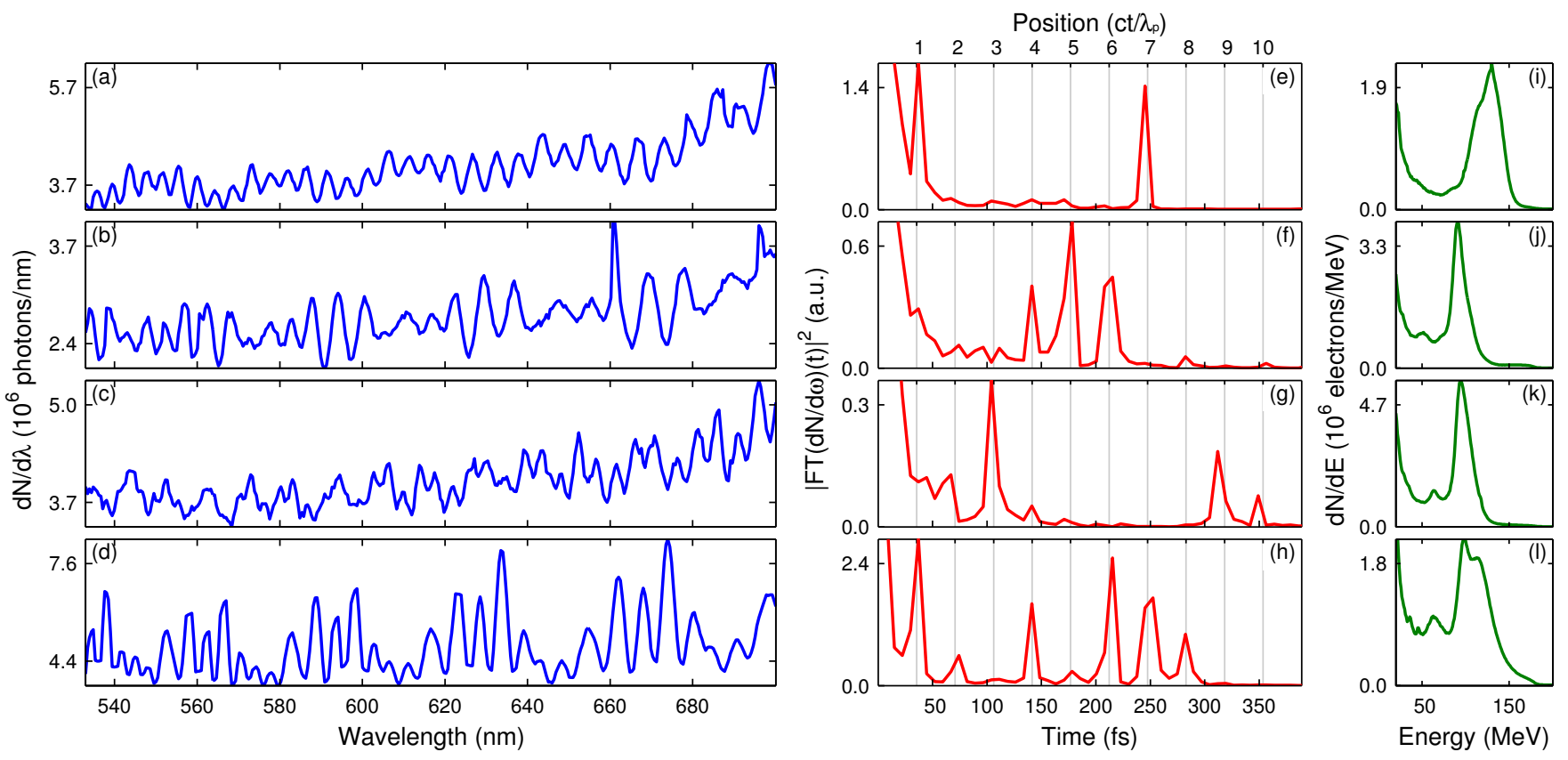

Figure 2. (Color online) Selected examples of complex CTR modulations, obtained using CPI for $n_{e}=10 \times 10^{18} \mathrm{~cm}^{-3}$ and $\lambda_{p}=10.6 \mu \mathrm{m}$. Three panels on each row represent one measurement. (a-d): CTR spectra. (e-h): Fourier transform of the CTR spectra. (i-l): Corresponding electron spectra.

lines in Fig. 1(h-n). The CTR spectra are sinusoidal and the Fourier Transforms contain a single significant peak. This suggests that, in these measurements, two electron bunches were accelerated in two plasma wave periods, separated by up to $9 \lambda_{p}$ (see Fig. 1n). The rightmost panels in Fig. 1 show electron spectra, measured on the same laser shot. For some measurements, the electron spectrum contains several peaks. However, no clear correlation between the observation of multiple components in the electron spectrum and spectral oscillations in the CTR spectrum was evident when considering the complete dataset. This is the first indication that one of the bunches contains most of the charge.

In some cases, the oscillations were more complex. Figure 2 shows some selected examples of such CTR spectra. For these examples, the Fourier transform contains several peaks. But, as in Fig. 1, each of these peaks is precisely centered on multiples of the plasma wavelength. Although the oscillations in the CTR spectrum change significantly from shot to shot, the peaks in the Fourier Transform are always centered on multiples of the plasma wavelength. These examples show that multiple electron bunches were trapped and accelerated in separate wave periods, at least ten plasma wave periods apart. However, as in Fig. 1, there is no consistent and clear signature of multiple components in the electron spectrum, indicating that a single bunch dominated the spectrum. Moreover, in Fig. 2g, there are Fourier peaks at $3 \lambda_{p}$ and $9 \lambda_{p}$. Interference between these two bunches should generate a peak at $6 \lambda_{p}$. The fact that this peak is absent in Fig. 2 $\mathrm{g}$ is another strong indication that the interferences are generated by a single, dominating pulse (presumably the first bunch, immediately behind the laser pulse), and the rest of the bunch train.

The depth of the modulations fluctuated from shot to shot, but had a tendency to be small when the modulation period was small, an indication that less charge was trapped far behind the laser pulse. The modulation depth of a function $f$ is described by the fringe visibility, $V=\left(f_{\max }-f_{\min }\right) /\left(f_{\max }+f_{\min }\right)$. Figure 3 shows the visibility of sinusoidal modulations that contained only one significant Fourier peak. These interferences were produced by only two electron bunches.

We now discuss the additional information that can be extracted from these spectral modulations. Consider two electron bunches separated by time $T$ and described by their normalized distribution $f_{1}(\vec{x})$ and $f_{2}(\vec{x})$. We assume that these electron bunches have a similar electron distribution and they radiate CTR which is collected through an angle $\theta_{0}>1 / \gamma$. The visibility of the spectral modulation is determined by the maximum and minimum values of the form factor $F(\omega)=$ $\left|q_{1} \hat{f}_{1}(\omega)+q_{2} \hat{f}_{2}(\omega)\right|^{2}$, where $\hat{f}_{i}(\omega)$ is the Fourier transform of $f_{i}(\vec{x})$, and $q_{i}$ is the charge of bunch $i$ :

$$
V(\omega)=\frac{F_{\max }-F_{\min }}{F_{\max }+F_{\min }}=\frac{2 \xi(\omega)}{1+\xi(\omega)^{2}}
$$

where $\xi(\omega)=\left(q_{2} / q_{1}\right) \hat{f}_{2}(\omega) / \hat{f}_{1}(\omega)$. For instance, for two gaussian pulses of RMS dura- 


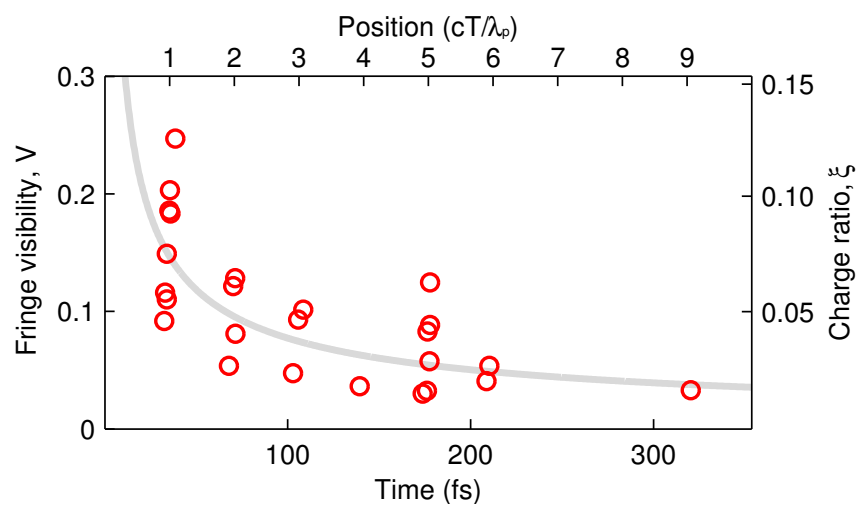

Figure 3. (Color online) Fringe visibility for sinusoidal oscillation that contained only one significant peak in the Fourier Transform, as function of the position of that peak. The charge ratio $\xi=q_{n} / q_{o}$ is calculated from the visibility using Eq. 2 in the limit where both bunches have similar shapes and energy distributions. The measurements were performed using CPI for $n_{e}=10 \times 10^{18} \mathrm{~cm}^{-3}$.

tion $\sigma_{1}$ and $\sigma_{2}$, and RMS radius $\sigma_{r 1}$ and $\sigma_{r 2}$, $\xi=\left(q_{2} / q_{1}\right) e^{\left(\sigma_{1}^{2}-\sigma_{2}^{2}\right) \omega^{2} / 2} e^{\left(\sigma_{r 1}^{2}-\sigma_{r 2}^{2}\right) \sin ^{2} \theta \omega^{2} / 2 c^{2}} . \quad$ Thus, the visibility depends on the charge ratio as well as on wavelength (through the bunch shapes). However, the experimental data in Fig. 1 and Fig. 2 clearly shows that the fringe visibility is nearly independent on wavelength (see also suppl. info. for a more detailed analysis [13]). From this, we can deduce that the dependence on $\hat{f}_{1}$ and $\hat{f}_{2}$ can be neglected, i.e. for gaussian bunches $\left(\sigma_{1}^{2}-\sigma_{2}^{2}\right) \omega^{2}+\left(\sigma_{r 1}^{2} / c^{2}-\sigma_{r 2}^{2} / c^{2}\right) \sin ^{2} \theta \omega^{2} \ll 1$. Physically, it means that both bunches have similar shapes. Thus, there is a simple relationship between the visibility and the charge ratio, which reduces in the limit $V^{2} \ll 1$ to $q_{2} / q_{1} \simeq V / 2$. Figure 3 shows the charge ratio as function of the position of the Fourier peak. The ratio is maximal at around $10 \%$, when the two pulses are separated by a single plasma wave period, and then decreases as the separation increases. This confirms the previously discussed indications that a single bunch contains the large majority of the total charge.

The measurements were performed using three plasma densities and employing either SI or CPI as methods for

Table I. Summary of the three datasets included in this study. The mechanism for injection is either self-injection (SI) or colliding pulse injection (CPI), depending on the plasma density, $n_{e}$ and the plasma wavelength, $\lambda_{p} . N_{t}$ is the number of measurements and $N_{m}$ is the number of measurements with modulations. $Q$ is the average charge and $T_{\max }$ is the maximum bunch separation in the dataset.

\begin{tabular}{ccccccc}
\hline \hline Mech. & $n_{e}\left(\mathrm{~cm}^{-3}\right)$ & $\lambda_{p}(\mu \mathrm{m})$ & $N_{t}$ & $N_{m} / N_{t}$ & $Q(\mathrm{pC})$ & $c T_{\max } / \lambda_{p}$ \\
\hline SI & $13 \times 10^{18}$ & 9.3 & 61 & $10 \%$ & 91 & 1.1 \\
CPI & $10 \times 10^{18}$ & 10.6 & 114 & $56 \%$ & 27 & 9.9 \\
CPI & $7 \times 10^{18}$ & 12.5 & 24 & $42 \%$ & 34 & 6.9 \\
\hline \hline
\end{tabular}

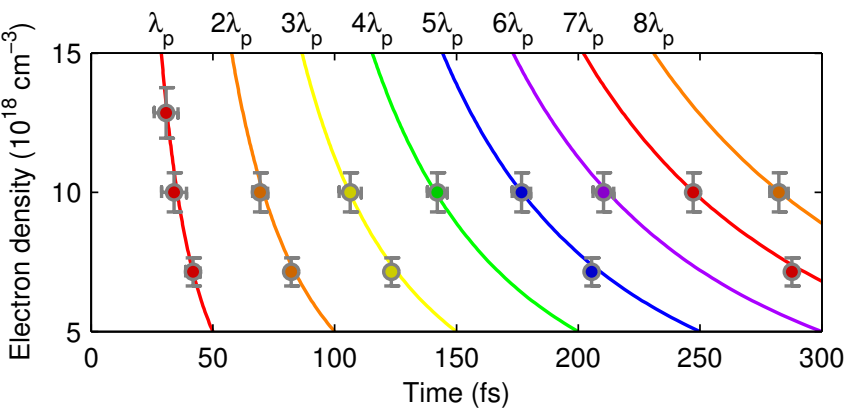

Figure 4. (Color online) Average positions of the Fourier peaks for different plasma densities (o) are compared to multiples of the plasma wave period (lines). Horizontal errorbars denote the standard deviation over the complete dataset. Vertical errorbars is an estimate of the uncertainty in plasma density.

injection. A summary of the measurements is shown in Table I. At the highest density $\left(13 \times 10^{18} \mathrm{~cm}^{-3}\right)$ and using SI, only $10 \%$ of the measurements produced oscillations in CTR spectrum, and Fourier peaks only occurred at separations of a single wave period. At lower densities, and using CPI, oscillations occurred on about $50 \%$ of the measurements and Fourier peaks occurred at separations up to 10 wave periods. For every measurement, the temporal positions $T_{n}$ of the Fourier peaks were determined. Each peak was classified as belonging to wave period $n$ if $\left|n-c T_{n} / \lambda_{p}\right|<0.5$ and the average position was then calculated over the complete dataset for each plasma density (for details, see suppl. info. [13]). The result from this analysis is shown in Fig. 4. For all three datasets, and for each peak, the average positions coincide with the positions of multiples of the plasma wavelength.

The measurements summarized in Table I suggest that CPI leads to longer bunch trains more often than SI. A possible reason for this could be the amount of injected charge: in the case of SI, the injected beam loads reaches more than $90 \mathrm{pC}$, i.e. three times more than in the CPI case. When the injected charge is large, beam loading damps the wakefield in the trailing buckets and trapping is more difficult. Thus, beam loading mitigates the injection of multiple beamlets. In the case of CPI, the injection is less than $30 \mathrm{pC}$ and the wakefield in the trailing buckets is not as damped, thus multiple injection is more likely to occur. Note that this is a very general trend in laser-plasma accelerators: large injected charge can prevent multiple injection through beam loading but usually results in larger energy spread and degraded beam quality [21]. On the other hand, smaller injected charge results in narrow energy spread but our measurements reveal also that multiple injection is more likely to occur.

In conclusion, we have shown that CTR is a powerful and simple diagnostic, that gives crucial information on the temporal distribution of the electron beam. The experimental data indicate clearly that multiple electron 
bunches can be produced and that they are separated by multiples of $\lambda_{p} / c$. Several experimental evidence indicate that a single electron bunch contains most of the charge. The possibility of producing one or several electron bunches shows another interesting feature of laser plasma accelerators. For the development of Free Electron Lasers, where the temporal structure of the electron bunches as well as the peak current are of prime importance, it appears that enhanced control over the injection mechanism and beam loading might become necessary.

We acknowledge the support from the European Research Council for the funding the ERC PARIS project (contract No. 226424). O.L. acknowledges additional support from the Swedish Research Council (including its Linneus grant to LLC).

* olle.lundh@fysik.lth.se

$\dagger$ jerome.faure@ensta-paristech.fr

[1] T. Tajima and J. M. Dawson, Phys. Rev. Lett. 43, 267 (1979).

[2] J. Faure, C. Rechatin, A. Norlin, A. Lifschitz, Y. Glinec, and V. Malka, Nature 444, 737 (2006).

[3] W. P. Leemans, B. Nagler, A. J. Gonsalves, C. Tóth, K. Nakamura, C. G. R. Geddes, E. Esarey, C. B. Schroeder, and S. M. Hooker, Nature Phys. 2, 696 (2006).

[4] O. Lundh, J. Lim, C. Rechatin, L. Ammoura, A. BenIsmail, X. Davoine, G. Gallot, J.-P. Goddet, E. Lefebvre, V. Malka, and J. Faure, Nature Physics 7, 219 (2011).

[5] A. Tremaine, J. B. Rosenzweig, S. Anderson, P. Frigola, M. Hogan, A. Murokh, C. Pellegrini, D. C. Nguyen, and R. L. Sheffield, Phys. Rev. Lett. 81, 5816 (1998).

[6] W. P. Leemans, C. G. R. Geddes, J. Faure, C. Tóth, J. van Tilborg, C. B. Schroeder, E. Esarey, G. Fubiani, D. Auerbach, B. Marcelis, M. A. Carnahan, R. A. Kaindl, J. Byrd, and M. C. Martin, Phys. Rev. Lett. 91, 074802
(2003).

[7] A. Buck, M. Nicolai, K. Schmid, C. M. S. Sears, A. Savert, J. M. Mikhailova, F. Krausz, M. C. Kaluza, and L. Veisz, Nat. Phys. 7, 543 (2011).

[8] J. van Tilborg, C. B. Schroeder, C. V. Filip, C. Tóth, C. G. R. Geddes, G. Fubiani, E. Esarey, and W. P. Leemans, Phys. Plasmas 13, 056704 (2006).

[9] Y. Glinec, J. Faure, A. Norlin, A. Pukhov, and V. Malka, Phys. Rev. Lett. 98, 194801 (2007).

[10] Y. Shibata, K. Ishi, T. Takahashi, T. Kanai, M. Ikezawa, K. Takami, T. Matsuyama, K. Kobayashi, and Y. Fujita, Phys. Rev. A 45, R8340 (1992).

[11] C. Schroeder, E. Esarey, and J. van Tilborg and W.P. Leemans, Phys. Rev. E 69, 016501 (2004).

[12] J. Faure, Y. Glinec, G. Gallot, and V. Malka, Phys. Plasmas 13, 056706 (2006).

[13] See Supplemental Material at [URL will be inserted by publisher] for details.

[14] E. Esarey, R. F. Hubbard, W. P. Leemans, A. Ting, and P. Sprangle, Phys. Rev. Lett. 79, 2682 (1997).

[15] G. Fubiani, E. Esarey, C. B. Schroeder, and W. P. Leemans, Phys. Rev. E 70, 016402 (2004).

[16] S. V. Bulanov, F. Pegoraro, A. M. Pukhov, and A. S. Sakharov, Phys. Rev. Lett. 78, 4205 (1997).

[17] J. Faure, Y. Glinec, A. Pukhov, S. Kiselev, S. Gordienko, E. Lefebvre, J.-P. Rousseau, F. Burgy, and V. Malka, Nature 431, 541 (2004).

[18] C. G. R. Geddes, C. Toth, J. van Tilborg, E. Esarey, C. B. Schroeder, D. Bruhwiler, C. Nieter, J. Cary, and W. P. Leemans, Nature 431, 538 (2004).

[19] S. P. D. Mangles, C. D. Murphy, Z. Najmudin, A. G. R. Thomas, J. L. Collier, A. E. Dangor, E. J. Divall, P. S. Foster, J. G. Gallacher, C. J. Hooker, D. A. Jaroszynski, A. J. Langley, W. B. Mori, P. A. Norreys, F. S. Tsung, R. Viskup, B. R. Walton, and K. Krushelnick, Nature 431, 535 (2004).

[20] C. Rechatin, J. Faure, A. Ben-Ismail, J. Lim, R. Fitour, A. Specka, H. Videau, A. Tafzi, F. Burgy, and V. Malka, Phys. Rev. Lett. 102, 164801 (2009).

[21] C. Rechatin, X. Davoine, A. Lifschitz, A. B. Ismail, J. Lim, E. Lefebvre, J. Faure, and V. Malka, Phys. Rev. Lett. 103, 194804 (2009). 